\title{
Air Pollution and its impacts on health in Africa - insights from the State of Global Air 2020
}

\author{
Joanna Keel, Katherine Walker, Pallavi Pant (10* \\ Health Effects Institute, 75 Federal Street, Suite 1400, Boston, MA, 02110 USA \\ *Corresponding Author E-mail: ppant@healtheffects.org
}

https://doi.org/10.17159/caj/2020/30/2.9270

According to the State of Global Air $2020^{1}$, total air pollution (including ambient $\mathrm{PM}_{2.5}$ and ozone as well as household air pollution) emerged as the 3rd leading risk factor for death and disability in sub-Saharan Africa in 2019, surpassed only by malnutrition and unsafe water, sanitation and hygiene. Exposure to air pollution was linked to more than 900,000 premature deaths (95\% uncertainty interval [UI]: 760,000-1.1 million) in sub-Saharan Africa, equal to about $12 \%$ (UI: 10\%-13\%) of all deaths in the region. Globally, air pollution was linked to 6.67 million deaths (UI: 5.90-7.49 million) in 2019.

Countries in Africa continue to experience some of the highest exposures to ambient $\mathrm{PM}_{2.5}$ in the world, along with countries in the Middle East and Asia. In both the sub-Saharan Africa and the North Africa and Middle East regions (as defined by the GBD project), the $\mathrm{PM}_{2.5}$ exposure was $\sim 44 \mu \mathrm{g} / \mathrm{m}^{3}$ (UI: $36-54 \mu \mathrm{g} / \mathrm{m}^{3}, 38$ $\left.50 \mu \mathrm{g} / \mathrm{m}^{3}\right)$ in 2019, severalfold higher than the $\mathrm{PM}_{2.5}$ Air Quality Guideline set at $10 \mu \mathrm{g} / \mathrm{m}^{3}$ by the World Health Organization. Within the region, Egypt, Niger, Nigeria, Mauritania, and Cameroon experienced even higher $\mathrm{PM}_{2.5}$ concentrations in 2019, ranging from $65 \mu \mathrm{g} / \mathrm{m}^{3}$ to $80 \mu \mathrm{g} / \mathrm{m}^{3}$. These countries all rank among the top 10 countries in the world with the highest levels of $\mathrm{PM}_{2.5}$. Note that higher $\mathrm{PM}_{2.5}$ exposures in some North African countries include contributions from local dust sources as well as wind-blown dust from the Sahara Desert.

Across Sub-Saharan Africa, $\mathrm{PM}_{2.5}$ exposures have increased by $3 \%$ since 2010, even as other regions including Southeast Asia, East Asia, and Oceania have seen reductions. Nigeria saw a $12 \%$ increase $\left(7.5 \mathrm{\mu g} / \mathrm{m}^{3}\right)$ over the same time period, from $63 \mu \mathrm{g} / \mathrm{m}^{3}$ (UI: $41-92 \mu \mathrm{g} / \mathrm{m}^{3}$ ) to $70 \mu \mathrm{g} / \mathrm{m}^{3}$ (UI: $45-105 \mu \mathrm{g} / \mathrm{m}^{3}$ ).

A major source of exposure and health impact in much of Africa continues to be linked to lack of access to clean energy. Without it, a large percentage of people rely on burning of various solid fuels (coal, charcoal, wood, agricultural residue) as well as kerosene for heating or for cooking using open fires or cookstoves with limited ventilation resulting in high exposures to particulate matter in their homes, referred to as household air pollution. In fact, more than $97 \%$ of the populations in Central African Republic, South Sudan, Rwanda, Burundi, Niger, Mali, Madagascar, Tanzania, Uganda, and Guinea-Bissau all relied on solid fuels for cooking in 2019.

Progress toward cleaner fuels has been slow in the region on average. However, some countries have made great strides in reducing reliance on solid fuels for cooking in the last decade. In Equatorial Guinea, the percentage of the population relying on solid fuels decreased from $50 \%$ (UI: $45-55 \%$ ) to $25 \%$ (UI: $18-33 \%$ ) between 2010 and 2019. In the same time period, Cabo Verde saw a decrease from 30\% (UI: 26-34\%) to 19\% (UI:15-24\%), and Botswana from 48\% (UI: $45-52 \%$ ) to $39 \%$ (UI: $30-48 \%$ ).

Exposure to both outdoor and indoor air pollution can each place a heavy burden on health, although the burden they impose is not equal. In Sub-Saharan Africa, exposure to household air pollution contributes to more than three times the number of deaths (684,000 deaths; UI: 516,000-863,000 deaths, 9\%; UI: $7-11 \%$ of all deaths in 2019) as exposure to ambient $\mathrm{PM}_{25}$ (216,000 deaths; UI: 142,000-311,000 deaths, about 3\%; UI: $2-4 \%$ of all deaths). $43 \%$ of air pollution deaths in sub-Saharan Africa result from non-communicable diseases that are linked with exposure to air pollution, including chronic obstructive pulmonary disease (COPD), diabetes, ischemic heart disease, lung cancer and stroke. Air pollution is also linked to lower respiratory infections, which pose a particular risk of illness and death to children under 5 years of age.

For the first time, State of Global Air also presented results on the impacts of air pollution on the health of infants in their first month of life. Babies born to mothers exposed to air pollution during pregnancy may be born too early or too small, which may result in a variety of additional complications including lower-respiratory infections, diarrheal diseases, brain damage

${ }^{1}$ The State of Global Air is a collaboration between the Health Effects Institute and the Institute for Health Metrics and Evaluation, based on annual data from the Global Burden of Disease (GBD) Study. Each year, the GBD study produces estimates of current and yearly trends in exposures and burden of disease from key risk factors in countries and territories around the world, making use of state-of-the-art comparative risk assessment methods.

${ }^{2}$ The amount of uncertainty differs from place to place given differences in the quantity and quality of the underlying data for the estimates.

${ }^{3}$ In much of Africa, data on air quality from ground-based monitors are limited. The numbers presented represent the best estimate of annual average exposures based on a combination of ground-based monitoring data, satellite data and modelled estimates. 


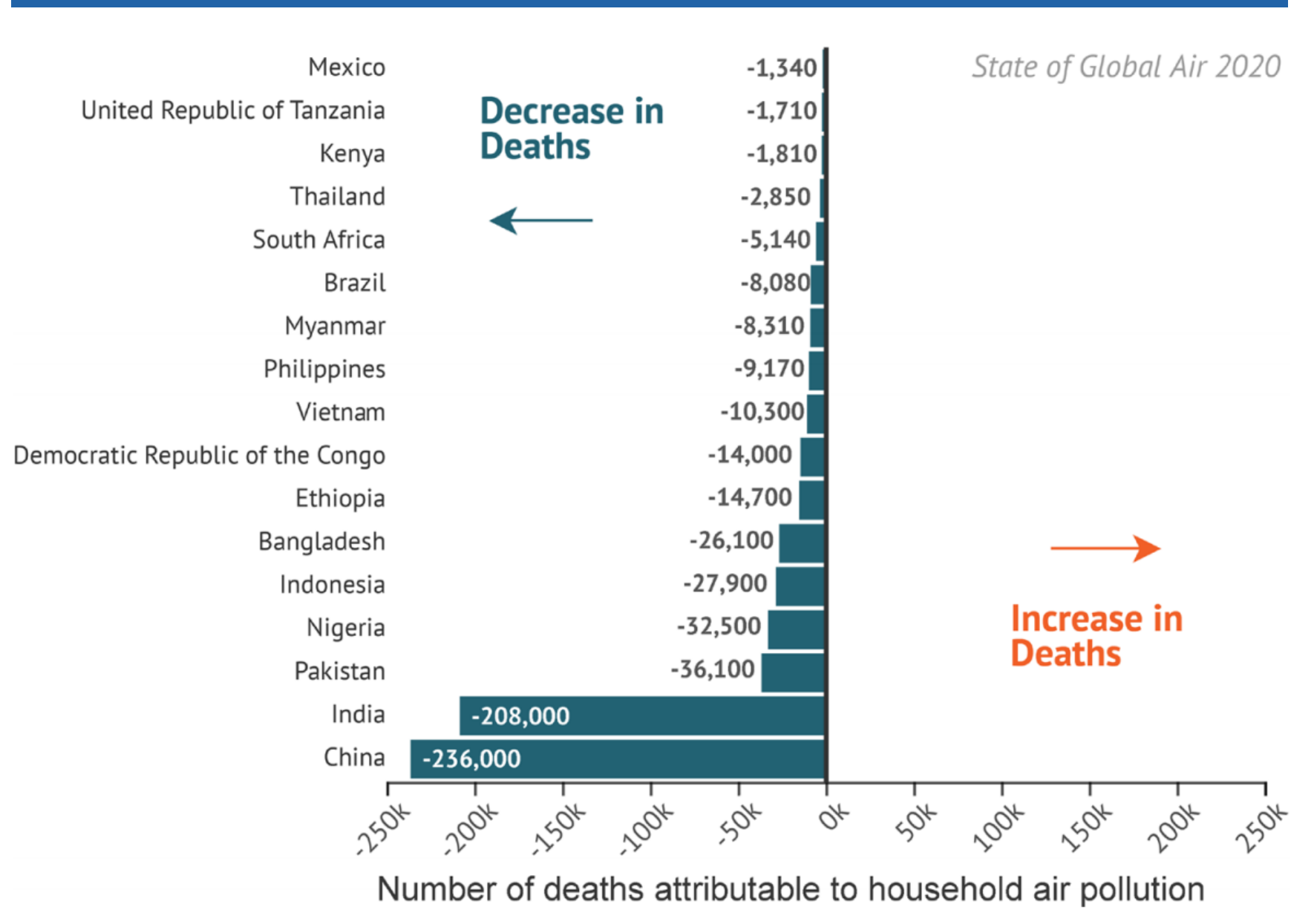

Change in the number of deaths attributable to household air pollution in the 17 countries with over 50 million people and at least $10 \%$ of their population cooking with solid fuels, 2010-2019.

Figure 1: Several countries in Africa have seen reductions in the number of deaths attributable to household air pollution in the last decade. Household air pollution has historically been and remains a challenge in other regions of the world as well.

and inflammation, blood disorders, and jaundice and in some cases, death. Sub-Saharan Africa has carried a particularly heavy burden for infants. In 2019, $23 \%$ (UI: $21-25 \%$ ) or more than 235,000 (UI: 191,000-291,000) infant deaths were attributable to air pollution in this age group, almost half the global total. Household air pollution is the major culprit in the region accounting for about $80 \%$ of these infant deaths.

Air pollution continues to impose a heavy and increasing burden on health in many countries in Africa. Millions of people every year have borne an avoidable burden from air pollution-related disease and premature mortality; the impacts on newborns and on children under five are particularly harsh. While exposures to $\mathrm{PM}_{2.5}$ and household air pollution remain high across the continent, some countries are beginning to undertake policy measures to improve air quality. A silver lining is the increasing adoption of cleaner cooking technologies in some countries across Africa, which is contributing to declines in the number of people exposed to household air pollution. By publishing these trends each year, we seek to focus public attention on the progress, but also on the major problems that have yet to be addressed. Our objective is to inform actions to reduce pollution in ways that have the greatest potential to benefit health.

The State of Global Air is a source of open-access, objective, high-quality, and comparable air quality and health data and information. Learn more at our website: https://www. stateofglobalair.org and contact us with any questions or opportunities for collaboration (contactsoga@healtheffects. org). Health Effects Institute released the fourth edition of its State of Global Air 2020 annual report and interactive website on the levels and trends of air quality and health in countries around the world on October 21, 2020. 